\title{
Cranial magnetic resonance imaging and school performance in very low birth weight infants in adolescence
}

\author{
R W I Cooke, L J Abernethy
}

\begin{abstract}
Aim-To determine whether neurological deficits are associated with structural anomalies of the brain in very low birthweight (VLBW) infants with subsequent learning disorders but without cerebral palsy, or whether other factors, such as poor early growth, are responsible.

Methods-Eighty seven VLBW infants and eight term controls who had been examined at school between the ages of 12 and 13 years, had cranial magnetic resonance imaging (MRI) scans at 15-17 years of age. Results-Thirty seven $(42.5 \%)$ of the VLBW children had abnormalities reported on their scans (two porencephaly, 28 periventricular leucomalacia, 24 ventricular dilatation, and 15 thinning of the corpus callosum). No significant differences in intelligence quotient, motor clumsiness, or frequency of attention deficit / hyperactivity disorder were observed between those children with MRI lesions and those with normal scans. Quantitative measurements showed the VLBW infants had smaller brains, and a relatively smaller corpus callosum compared with controls. No association between brain measurements and school performance was observed among the VLBW infants. Conclusions-The difficulties experienced by VLBW children at school are unlikely to be the result of perinatal brain injury, but they might to be attributable to the effects of poor postnatal growth.
\end{abstract}

(Arch Dis Child Fetal Neonatal Ed 1999;81:F116-F121)

Keywords: very low birthweight; learning disorder; MRI; periventricular leucomalacia

The survival of very preterm infants has improved considerably in recent years, leading to an increased interest in their subsequent intellectual and functional development. Although $10-15 \%$ of survivors weighing less than $1500 \mathrm{~g}$ at birth have a major physical disability identified in the preschool period, most are initially perceived as normal and enter mainstream schools. ${ }^{1}$ Studies of these children have, however, shown that as many as 35\% experience learning difficulties in one or more subjects, and that these difficulties are predicted by the presence of minor motor clumsiness at an earlier age. ${ }^{2}$ This clumsiness has been ascribed by some to a maturational delay, but by others to early cerebral injury. Longitudinal studies of children with motor clumsiness tend to show improvement with time, although problems persist. ${ }^{3}$ Associated problems such as specific learning disabilities, attentional, and behavioural disorders become more prominent.

Early attempts to correlate brain imaging, such as cranial ultrasonography, with subsequent outcome in VLBW infants were successful for major impairments such as cerebral palsy, but not as good for minor clumsiness and learning disorders. ${ }^{45}$ This could be due in part to the limitations of early ultrasound scanning, or possibly the action of other early environmental factors on the child as s/he develops. More recent ultrasound studies have shown some association with more minor disorders. ${ }^{6}$

In recent years magnetic resonance imaging (MRI) has shown that it can define cerebral anatomy with a precision not previously available, and with minimal invasiveness. Such scans can identify minor structural changes related to cerebral atrophy or delayed myelination of tracts secondary to perinatal or later insults. ${ }^{7}$ Although most MRI studies on young children have concentrated on lesions and myelination defects which predict cerebral palsy and visual defects, less severe outcomes have also been studied. In children with sickle cell disease the presence of minor infarcts detected on MRI correlated well with poorer performance on the Movement ABC, a test of motor impairment. ${ }^{8}$ Differences in the sizes of the corpus callosum and of the caudate nuclei have been seen in children with attention deficit or learning disorders, but born at term. ${ }^{9-14}$ The corpus callosum is very vulnerable in sick VLBW infants, and is often the site of cystic leucomalacia or atrophy, particularly posteriorly. The role of the corpus callosum is thought to relate to interhemispheric function and coordination, allowing the two cerebral hemispheres to share learning and memory. The posterior corpus callosum has a role in visual discrimination. Its fibres comprise $11 \%$ of the supratentorial brain tissue. The caudate nuclei receive inputs from cortical regions implicated in executive functioning and attentional tasks. Damage to the structure or myelination of either may result in minor motor dysfunction, attention deficit, or learning disability.

This study was designed to test the hypothesis that poor learning, attention deficit, and minor motor deficits in VLBW infants at school were related to evidence for perinatal brain injury on cerebral MRI scans. 


\section{Methods}

A cohort of 137 VLBW infants attending mainstream schools was studied in detail up to the age of 13 years. ${ }^{3}$ They had been followed up prospectively from birth, with formal examinations at school at 6 and 13 years. Detailed information was available on health and growth status, and the perinatal period. IQ was measured (Wechsler Intelligence Scale for Children III) and motor disability assessed using the Movement ABC. Learning difficulties were assessed using the Suffolk Reading Scale, the Basic Mathematics Test, and the SPAR spelling test. Teacher and student questionaries were also used. Psychiatric assessments were also performed for anxiety, fears, depression, antisocial behaviour and self esteem. Parents and teachers also completed Rutter A and B questionaries with Connor's Hyperactivity Scale. A Child and Adolescent Psychiatric Assessment (CAPA) interview was conducted with the parent and child. ${ }^{14}$ Visual function studies of acuity, stereopsis, and contrast sensitivity were done. All these data are available on a computed database, together with extensive demographic and medical data, and have been reported in detail before. ${ }^{315}$ Only the IQ scores, Movement ABC scores, and the presence of attention deficit at 13 years were used in this study. Attention deficit included attention deficit disorder, hyperactivity disorder, or attention deficit/hyperactivity disorder diagnosed from the CAPA interviews. The disorders were required to be sufficient to cause functional impairment at school or socially. The Movement ABC scores were originally scored out of a total of 40 , but were recalculated to facilitate comparison with similar test results performed at 6 and 8 years which were scored out of 16 .

Those children living within an hour's journey from Liverpool were invited by letter to attend for an MRI scan. Full explanations of the purpose and nature of the study were given. The subjects were between 15 and 17 years of age at the time of MRI scan. They were not paid, but travel expenses were met in full. No sedation was used. MRI brain scans were performed using a 0.5 Tesla Philips Gyroscan T5-NT scanner (Philips Medical Systems, Best, Netherlands).

The following sequences were used on all subjects: sagittal $\mathrm{T} 1$ weighted spin echo ( $\mathrm{TR}=387, \mathrm{TE}=20)$; axial $\mathrm{T} 1$ weighted spin echo $(\mathrm{TR}=475$, $\mathrm{TE}=25)$; axial fluid attenuated inversion recovery (FLAIR) ( $\mathrm{TR}=6000$, $\mathrm{TE}=150, \mathrm{~T} 1=2000)$; coronal $\mathrm{T} 1$ weighted gradient echo, volume acquisition $(\mathrm{TR}=200$, $\mathrm{TE}=30, \mathrm{TI}=13$, flip angle=30); coronal $\mathrm{T} 2$ weighted fast spin echo ( $T R=3000, T E=120)$.

Images were viewed as film copy and on an Easivision workstation, and were reported by a paediatric radiologist (LA) who was experienced in paediatric neuroimaging, and unaware of the clinical status of the individual subjects and of the educational and psychometric test results.

Scans were reported as showing evidence of thinning of the corpus callosum (fig 1), ventricular enlargement (fig 2), periventricular leucomalacia (fig 2), porencephaly, (fig 3) or none of these. Periventricular leucomalacia was recognised by a characteristic triad of abnormalities; abnormally high signal within the periventricular white matter on $\mathrm{T} 2$-weighted and FLAIR images, loss of periventricular white matter, particularly in the periatrial regions, and compensatory focal ventricular enlargement adjacent to regions of abnormal signal intensity. ${ }^{16}$ Porencephaly was recognised as a well defined cavity within the brain substance, communicating with the lateral ventricle and containing fluid identical with that of cerebrospinal fluid.

Measurements were made of the overall length of the corpus callosum, the cross sectional areas of the sagittal, left and right transverse and coronal sections of the brain, the cross sectional area of the corpus callosum (entire and as anterior, middle, and posterior parts), and the transverse and coronal mid cross sectional areas of the right and left caudate nuclei. The cross sectional area of the corpus callosum as a percentage of the sagittal sectional area of the brain, and the transverse
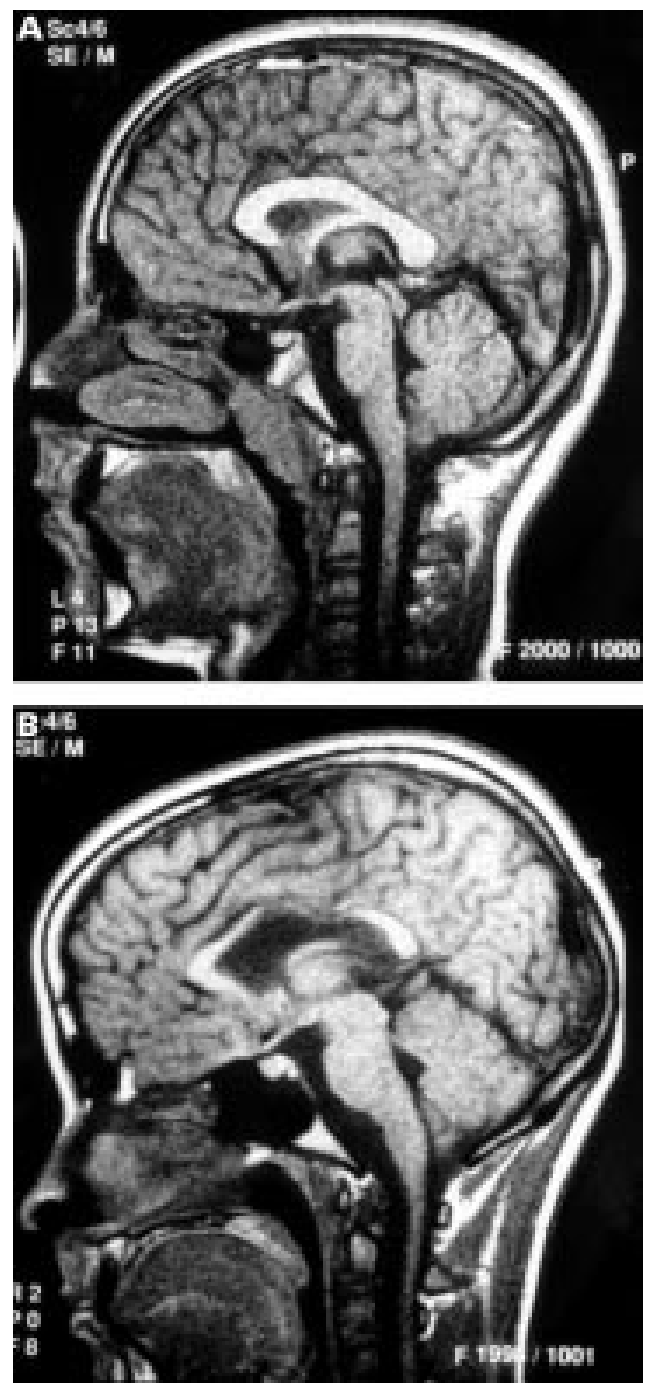

Figure 1 Sagittal T1 weighted spin echo MR images of the brain $(T R=387 \mathrm{~ms}, T E=20 \mathrm{~ms})$ : $(A)$ normal corpus callosum; (B) noticeable thinning of corpus callosum, particularly in middle section. 

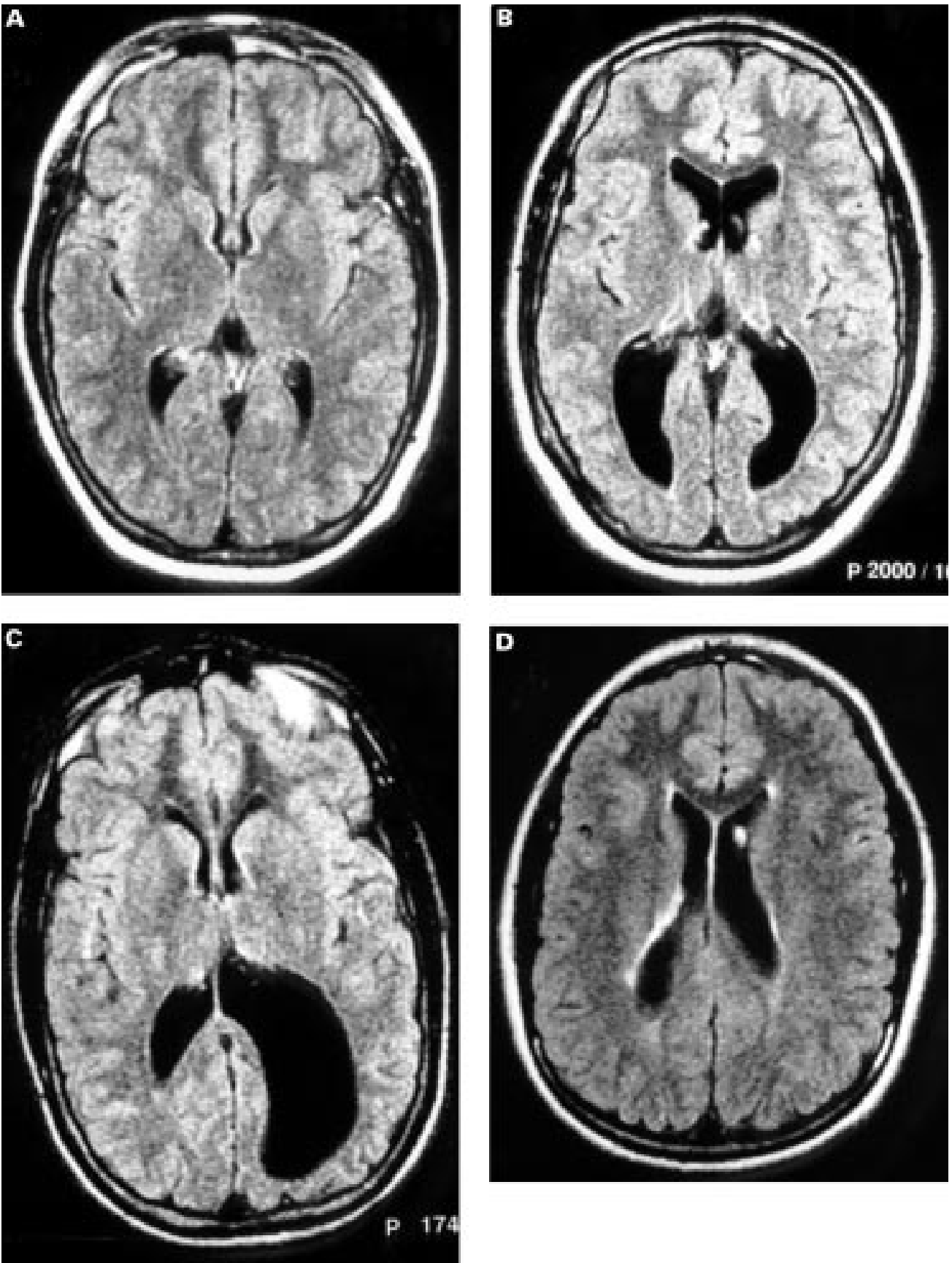

Figure 2 Axial fluid attenuated inversion recovery magnetic resonance images of the brain $(T R=6000 \mathrm{~ms}, T I=20000$ ms): (A) normal; (B) ventricular dilatation with loss of periventricular white matter; $(C)$ asymmetrical dilatation of the occipital horn of the left lateral ventricle, with loss of periventricular white matter; (D) high signal within the periventricular white matter, indicating periventricular leucomalacia.

and axial areas of the right and left caudate nuclei as a percentage of the transverse and axial sectional areas of the respective cerebral hemispheres, were also calculated.

The study was approved by the Regional Paediatric Research Ethics Committee.

\section{Results}

One hundred and ten children from the original cohort who lived within an hour's journey time of the MRI centre were invited to take part in the study: 96 replied and 87 were eventually scanned. Reasons for not being scanned in those who replied included four refusals, one pregnancy, one technical failure and two who failed to attend. Twenty six of the original control children were also approached, and eight of these agreed to be scanned. A high proportion of the subjects wore dental braces, which, in some instances, interfered with the scan quality.

The 87 index children had been born at a mean gestational age of 28.6 (1.7) weeks (range 24-35 weeks) and had a mean birthweight of 1103 (203) g (range 630-1500 g). Seventeen were twins and nine were below the 10 th percentile of weight for gestational age. Forty one had received antenatal steroids and eight had shown fetal distress intrapartum. There were 47 girls and 40 boys; 57 received respiratory support from 1 to 88 days; 19 had 

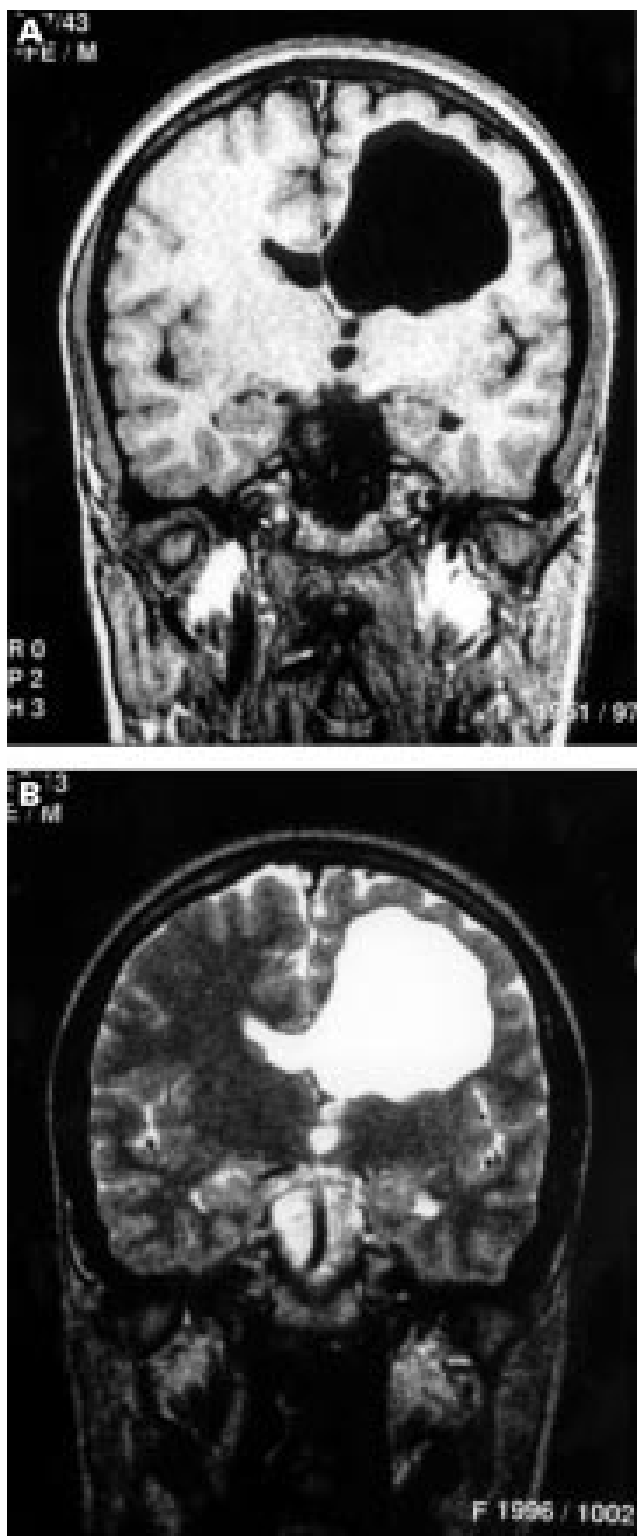

Figure 3 (A) Coronal T1 weighted gradient echo $M R$ image $(T R=200 \mathrm{~ms}, T E=30 \mathrm{~ms}, T I=13 \mathrm{~ms}$, Flip angle of 30 degrees; (B) coronal T2 weighted fast spin echo $M R$ image $(T R=30000 \mathrm{~ms}, T E=120 \mathrm{~ms})$. Porencephalic cyst formation adjacent to the frontal horn of the left lateral ventricle.

ultrasound scan evidence of periventricular haemorrhage, which in four involved the brain parenchyma, and two subsequently had minor cystic leucomalacia. Twenty two had a positive blood culture and seven frank convulsions in the neonatal period. All the control subjects (four boys and four girls) had been born at term and had had a normal perinatal period.

Thirty seven $(42.5 \%)$ of the index children had one or more abnormalities reported on their scans. Twenty eight had periventricular leucomalacia of some degree, 24 had ventricular dilatation (usually asymmetrically), 15 had thinning of the corpus callosum (mainly posterior part). Two had porencephaly, but also had other abnormalities, and were not considered separately. None of the control children had any abnormalities on MRI scanning. Attention deficit/hyperactivity disorder had been ob-
Table 1

\begin{tabular}{|c|c|c|c|}
\hline & $A D H L$ & $A B C$ & $F S I Q$ \\
\hline MRI abnormality (37) & 8 & $\begin{array}{l}4 \\
(0-10)\end{array}$ & $\begin{array}{l}88 \\
(6-130)\end{array}$ \\
\hline No MRI abnormality (50) & 11 & $\begin{array}{l}3 \\
(0-12.5)\end{array}$ & $\begin{array}{l}88 \\
(48-137)\end{array}$ \\
\hline Thinning of corpus callosum (15) & 1 & $\begin{array}{l}5 \\
(1-9)\end{array}$ & $\begin{array}{l}87 \\
(64-130)\end{array}$ \\
\hline No thinning of corpus callosum (72) & 18 & $\begin{array}{l}3.5 \\
(0-12.5)\end{array}$ & $\begin{array}{l}89 \\
(48-137)\end{array}$ \\
\hline Ventricular dilatation (24) & 5 & $\begin{array}{l}4 \\
(0-8)\end{array}$ & $\begin{array}{l}87 \\
(56-130)\end{array}$ \\
\hline No ventricular dilatation (63) & 14 & $\begin{array}{l}3.5 \\
(0-12.5)\end{array}$ & $\begin{array}{l}88 \\
(48-137)\end{array}$ \\
\hline Periventricular leucomalacia (28) & 4 & $\begin{array}{l}5.25 \\
(0-10)\end{array}$ & $\begin{array}{l}87 \\
(56-130)\end{array}$ \\
\hline No periventricular leucomalacia (59) & 15 & $\begin{array}{l}3 \\
(0-12.5)\end{array}$ & $\begin{array}{l}89 \\
(48-137)\end{array}$ \\
\hline
\end{tabular}

ADHD: attention deficit/hyperactivity disorder; ABC: score from movement ABC; FSIQ: score from full scale IQ test. Numbers or median (range) for scores.

Table 2

\begin{tabular}{llll}
\hline & Cases $(n=87)$ & Controls $(n=8)$ & p Value \\
\hline CCL & $71(5)$ & $74(2)$ & 0.026 \\
CCANT & $238(37)$ & $282(22)$ & 0.001 \\
CCMID & $130(24)$ & $161(30)$ & 0.008 \\
CCPOST & $191(43)$ & $237(25)$ & 0.002 \\
CCTOT & $559(90)$ & $680(51)$ & 0.001 \\
SAGXS & $9838(889)$ & $10441(621)$ & 0.038 \\
CCPCT & $5.7(0.97)$ & $6.5(0.73)$ & 0.013 \\
LCAUDCS & $79(16)$ & $99(26)$ & 0.028 \\
LCAUDCSPCT & $1.71(0.35)$ & $2.00(0.67)$ & 0.220 \\
RCAUDCS & $85(15)$ & $102(26)$ & 0.088 \\
RCAUDCSPCT & $1.81(0.32)$ & $2.03(0.54)$ & 0.420 \\
LCAUDTS & $138(23)$ & $147(16)$ & 0.230 \\
LCAUDTSPCT & $1.76(0.32)$ & $1.65(0.17)$ & 0.340 \\
RCAUDTS & $138(28)$ & $153(22)$ & 0.082 \\
RCAUDTSPCT & $1.77(0.37)$ & $1.73(0.27)$ & 0.780 \\
CAUDCS ratio & $1.08(0.20)$ & $1.03(0.13)$ & 0.600 \\
CAUDTS ratio & $1.01(0.16)$ & $1.05(0.09)$ & 0.290 \\
LTCS & $4658(356)$ & $5004(278)$ & 0.005 \\
RTCS & $4688(344)$ & $5052(230)$ & 0.003 \\
LTTS & $7855(608)$ & $8904(367)$ & 0.001 \\
RTTS & $7805(599)$ & $8895(391)$ & 0.001 \\
\hline
\end{tabular}

CCL: length corpus callosum (mm); CCANT: anterior part of corpus callosum; CCMID: middle part of corpus callosum; CCPOST: posterior part of corpus callosum; CCTOT: whol of corpus callosum; SAGXS: saggital section of brain; CCPCT: area of corpus callosum as percentage of sagitta section of brain; LCAUDCS: left caudate nucleus in mid-coronal section; LCAUDCSPCT: left caudate nucleus in mid-coronal section as percentage of left coronal cerebral hemisphere; RCAUDCS: right caudate nucleus in mid-coronal section; RCAUDCSPCT: right caudate nucleus in mid-coronal section as percentage of right coronal cerebral hemisphere; LCAUDTS: left caudate nucleus in mid-transverse section; LCAUDTSPCT: left caudate nucleus in mid-transverse section as percentage of left transverse cerebral hemisphere; RCAUDTS: right caudate nucleus in mid-transverse section; RCAUDTSPCT: right caudate mid-transverse section; RCAUDT
nucleus in mid-transverse section as percentage of right nucleus in mid-transverse section as percentage of right
transverse cerebral hemishere; CAUDCS ratio: ratio of right to transverse cerebral hemishere; CAUDCS ratio: ratio of right to
left coronal sections of caudate nuclei; CAUDTS ratio: ratio of left coronal sections of caudate nuclei; CAUDTS ratio: ratio
right to left transverse sections of caudate nuclei; LTCS: left right to left transverse sections of caudate nuclei; LTCS: left
coronal section cerebral hemisphere; RTCS: right coronal coronal section cerebral hemisphere; RTCS: right coronal
section cerebral hemisphere; LTTS: left transverse section cerebral hemisphere; RTTS: right transverse section cerebral hemisphere.

served in 19 of the scanned index children. This was similarly distributed between those with MRI abnormalities and those without. Movement ABC scores and full scale IQ scores had similar medians and ranges in those with MRI changes or none (table 1). None of the controls had ADHD, high Movement ABC scores, or low IQ scores.

The linear and cross sectional area measurements were significantly smaller in the brains of index children than in the controls (table 2). The cross sectional area of the corpus callosum was significantly smaller in index children when corrected for the smaller brain area over- 
all (CCPCT), although the cross sectional areas of the right and left caudate nuclei were not, when treated in the same way (table 2). The ratios of right to left caudate cross sectional areas were similar in cases and controls. When all these measurements were compared in index children with and without ADHD, with a Movement ABC above and below 4, and an IQ above and below 88, no significant differences were seen for any of these measurements or ratios.

\section{Discussion}

Longer term follow up studies of infants born prematurely, but free of major neuromotor or sensory impairments, have almost invariably shown that a proportion of these children have behavioural and learning difficulties at school age. $^{231718}$ These difficulties have often been shown to occur in the presence of minor motor clumsiness or dyspraxia. The reasons for this high prevalence of problems have not been adequately explained. Social factors, poor nutrition or growth, and perinatal brain injury have all been suggested.

In the previous reports of our studies with this cohort of infants, ${ }^{23} 19$ class mate controls were used, who were a good match on several socioeconomic variables, yet the index children as a group performed substantially less well than the control group. Evidence for perinatal brain injury among the preterm children was clear in that $42.5 \%$ of them had MRI signs of periventricular leucomalacia, ventricular dilatation, or thinning of the corpus callosum. This figure is similar to that seen in children with overt cerebral palsy. ${ }^{7}$ However, the lack of a correlation between MRI appearances and IQ, motor coordination scores, and attention deficit/hyperactivity disorder seems to imply that another factor is more important in determining the high prevalence of these difficulties. Olsen et $a l^{20}$ found that $32 \%$ of a cohort of 8 year olds born prematurely had MRI evidence for periventricular leucomalacia, but that it occurred with equal frequency in those with minor motor dysfunction and in those considered to be clinically normal.

When index children and controls were examined together at 12 years, both height and head circumference were significantly correlated with IQ and ABC scores, in that poorer growth was associated with poorer scores. ${ }^{21}$ Quantitative methods applied to these MRI scans showed that the index population had smaller brains than term controls, and that the corpus callosum was significantly smaller as a percentage of brain size. Nevertheless, no significant differences were seen in the quantitative measurements when these were compared between cases with or without ADHD, motor clumsiness, or lower IQ. The absence of association between learning difficulties and measurable differences in brain MRI scans in these preterm infants differs from reported experience with term infants. ${ }^{9-14}$ This may indicate differing aetiologies for learning and minor motor problems in these two groups. For instance, in term infants the usual right/left caudate predominance is reported to be lost in
ADHD, but in our cases it remained unchanged. ${ }^{10}$ Reduction in the cross sectional area of the corpus callosum in term infants has been reported in term infants with learning difficulties, ${ }^{914}$ but although we observed a smaller corpus callosum in the preterm infants, this did not relate to learning or motor problems. More subtle deficits undetected by MRI may be responsible for poorer performance in these children.

The period between birth and term is one of relative malnutrition for many very preterm infants, because of acute and chronic illness and poor feed tolerance. Many fall below the third percentile by term, which is the time of maximum brain growth velocity in infants, and when vulnerability is at its highest. Improvement in nutrition at this critical period may contribute to improved school performance and growth in this high risk population.

This study was supported by the Medical Research Council (Grant Number G9615155).

The data concerning qualitative MRI in this paper were presented at the Spring Meeting of The Royal College of Paediatrics and Child Health in York, 1998.

We thank Mrs G Hughes and Mrs D Garlick, Mrs J Glynn, and Mrs H Tyrer at the MRI Unit, Royal Liverpool Children's Hospital (Alder Hey) for their skill and patience in performing the MRI scans, and the parents and children who gave their time to help in this study.

1 Escobar GJ, Littenburg B, Pettiti DB. Outcome among surviving very low birthweight infants: a meta-analysis. Arch viving very low birthweight
Dis Child 1991;66:204-11.

2 Marlow N, Roberts BL, Cooke RWI. Outcome at 8 years for children of birthweights of $1250 \mathrm{~g}$ or less. Arch Dis Child children of birth

3 Powls A, Botting N, Cooke RWI, Marlow N. Motor impairment at 12-13 years in children with a birthweight of less than 1250 g. Arch Dis Child Fetal neonatal ed 1995;72:F62-6.

4 Cooke RWI. Early and late ultrasonographic appearances and outcome in very low birthweight infants. Arch Dis Child 1987;62:931-7.

5 Levene MI, Dowling S, Graham M, Fogelman K, Galton $M$. Impaired motor function (clumsiness) in 5 year old children correlates with neonatal ultrasound scans. Arch Dis Child 1992;67:687-90.

6 Ringelberg J, van de Bor M. Outcome of transient periventricular echodensities in preterm infants. Neuropediatrics 1993;24:169-273.

7 de Vries L, Eken P, Groenendaal F, van Haastert IC, Meiners LC. Correlation betwen the degree of periventricular leukomalacia diagnosed using cranial ultrasound and MRI later in infancy in children with cerebral palsy. Neuropedilater in infancy in child
atrics $1993 ; 24: 263-8$.

atrics 1993;24:263-8.
9 Giedd JN, Castelanos FX, Casey BJ, et al. Quantitative morphology of the corpus callosum in attention deficit hyperactivity disorder. Am f Psychiatr 1994;151:665-9.

8 Mercuri E, Faundez JC, Roberts I, et al. Neurological "soft" signs may identify children with sickle cell disease who are at risk for stroke. Eur $\mathcal{F}$ Pediatr 1995;154:150-6.

10 Hynd GW, Semrud-Clikeman M, Lorys AR, Novey ES, Eliopulos D, Lyytinen H. Corpus callosum morphology in attention deficit-hyperactivity disorder: morphometric analysis of MRI. $\mathcal{F}$ Learn Disab 1991;24:141-6.

11 Castellanos FX, Geidd JN, Eckburg P, et al. Quantitative morphology of the caudate nucleus in attention deficit

12 Hynd GW, Hern KL, Novey ES, et al. Attention deficit/hyperactivity disorder and asymmetry of the deficit/hyperactivity disorder and asymmetry
caudate nucleus. 7 Child Neurol 1993;8:339-47.

13 Hynd GW, Semrud-Clikeman M, Lorys AR, Novey ES, Elopulos D. Brain morphology in developmental dyslexia and attention deficit disorder/hyperactivity. Arch Neurol 1990;47:919-26.

14 Njiokiktjien C, de Sonneville L, Vaal J. Callosal size in children with learning disabilities. Behav Brain Res 1994;64:213-18.

15 Angold A, Prendergast M, Cox A, Harrington R, Simonoff E, Rutter M. The Child and Adolescent Psychiatric Assessment. Psychological Medicine 1995;25:739-53.

16 Botting N, Powls A, Cooke RWI. Attention deficit hyperactivity disorders and other psychiatric outcomes in very low birthweight children at 12 years. $f$ Child Psychol Psychiatry 1997;38:931-41.

17 Drillien CM. The incidence of mental and physical handicaps at school age in children of very low handicaps at school age in child
birthweight. Pediatrics 1961;27:452-6.

18 Nickel RE, Bennett FC, Lamson FN. School performance of children with birthweights of $1000 \mathrm{~g}$ or less. Am $\mathcal{F}$ Dis Child 1982;136:105-9. 
19 Marlow N, Roberts BL, Cooke RWI. Motor skills in extremely low birthweight children at age 6 years. Arch Dis Child 1989;64:839-47.

20 Olsen P, Paakko E, Vainionpaa L, Pyhtinen J, Jarvelin MR. Magnetic resonance imaging of periventricular leukomalacia and its clinical correlation in children. Ann Neurol 1997;41:754-61.

\section{Commentary}

Long term follow up studies of very preterm infants without cerebral palsy show a high incidence of certain patterns of cognitive impairment. Ultrasound studies have suggested that there is an association between white matter echodensities and ventricular enlargement and subsequent cognitive impairments. ${ }^{1}$ Cooke and Abernethy's study, of a group of children who were born prematurely and attending mainstream schools, take these investigations further. They show MRI evidence of periventricular leucomalacia in $42.5 \%$ of the children, but no association between PVL and attention deficit disorder, low IQ, or minor motor impairment.

The authors suggest that inadequate nutrition, not hypoxia-ischaemia, may be the basis of these subsequent problems. We should also consider the effects of potentially toxic drugs such as steroids, the cessation of maternal hormones and maternal trophic factors, chronic hypoxia, abnormal environmental stimuli, and sepsis. Very preterm infants may be exposed to many or all of these factors between birth and term, a period during which there is a considerable increase in brain size and in maturation of cortical folding.

Recent studies have shown a high incidence of antenatal infection in preterm delivery, which may also lead to changes consistent with PVL - so-called "perinatal teloleucoencephalopathy"-in the developing white matter. ${ }^{2}$ These abnormalities reflect a more diffuse and milder abnormality than that found in PVL, but may not necessarily share the same aetiology.

Subsequent clinical impairment may result from early abnormalities in the developing brain that are difficult to detect on imaging studies performed in childhood, even with the use of quantification, as in Cooke's and Abernethy's study. Very preterm infants can now be serially imaged, and detail within the developing white matter, such as the presence of migrating cells, identified. ${ }^{34}$ In a cohort of very preterm infants subtle abnormalities
21 Powls A, Botting N, Cooke RWI, Marlow N. Growth impairment in very low birthweight children at 12 years: impairment in very low birthweight children at 12 years: Child 1996;75:F152-7.

22 Baker Fetal Neonnatal LL, Stevenson DK, Enzmann DR. End-stage periventricular leucomalacia: MR evaluation. Radiology 1988;168:809-15.

within the white matter have been detected on early MRI. ${ }^{5}$ These changes were not typical of classic PVL, but were often associated with the development of ventricular dilatation and widening of the extra cerebral space, suggestive of brain atrophy. Interestingly, in Cooke's and Abernethy's study, the preterm children had significantly smaller brains than the controls.

The study was unable to confirm previous MRI findings in children born at term with attention deficit disorder, such as changes in caudate size. With current technology, there may be no neuroimaging correlate for specific learning difficulties in children who are born preterm. It would be convenient if these cognitive disorders were simply related to measurable structural abnormalities within the cortex, such as delayed folding or reduced surface area. The reality, however, is that the brain is not simply the sum of its structures. These are richly interconnected, and the quantity of connections is difficult to measure-the quality even more so.

For the time being, we remain in a situation where we are seeking not only the aetiology of the "lesion," but also trying to locate the "lesion" itself.

MARY RUTHERFORD

Department of Paediatrics and Neonatal Medicine

Robert Steiner Magnetic Resonance Unit

Imperial College School of Medicine

Hammersmith Hospital

London W12 OHS

1 Guzzetta F, Shackelford GD, Volpe S, Perlman JM, Volpe JJ. Periventricular intraparenchymal echodensities in the premature newborn: critical determinant of neurologic outcome. Pediatrics 1986;78:995-1006.

2 Dammann O, Leviton A. Infeciton remote from the brain, neonatal white matter damage, and cerebral palsy in the preterm infant. Semin Pediatr Neurol 1998;5:190-201.

3 Batttin M, Maalouf E, Counsell S, et al. Magnetic resonance imaging of the brain in very preterm infants: visualization of the germinal matrix, early myelination, and cortical folding. Pediatrics 1998;101:957-62.

4 Childs MA, Ramenghi L, Evans D, et al.MR features of developing periventricular white matter in preterm infants: evidence of glial cell migration. Am f Neuroradiol 1998; 19 : 971-6.

5 Maalouf EF, Duggan PJ, Rutherford MA, et al. Magnetic resonance imaging of the brain in extremely preterm infants: normal and abnormal findings from birth to term. 7 Pediatr (in press). 